\title{
AUDITING AND COMPETITIVE BIDDING IN THE PUBLIC SECTOR
}

\author{
GERVAN FEARON AND LUTZ-ALEXANDER BUSCH
}

\begin{abstract}
This paper investigates the impact of a ministry's budget size on the choice between auditing a (Niskanen) bureau and employing competitive bidding in the provision of a publicly funded good. The ministry's marginal expected payoff increases fastest with budget size for auctioning as opposed to auditing. However, the ministry is shown to switch from a purely public provision to a competitive provision as the budget size increases even if expected fixed costs favor the audit. The study contributes to the literature by extending the Niskanen framework and by endogenizing the institutional arrangements for the provision of public sector goods.
\end{abstract}

Keywords: Niskanen bureau, public good provision, mechanism design, audit, auction.

JEL: H11, H4, D44, D73

\section{INTRODUCTION}

Just as the private sector, in recent years governments have looked towards outsourcing and privatization for the provision of goods and services by the public sector (De Fraja, 1993; Feenstra and Hanson, 1996). An increasing number of prison facilities in the USA are operated by private contractors and reportedly have 10 percent lower costs than their public sector counterparts (Hart et al. (1997).) Significant cost advantages for privatization over public provision have been found for refuse collection services in cities with 50,000 or more residents, while no significant difference was found for cities with fewer residents (Stevens (1978), Savas (1977).) For policing, it has been found that cities with a population of 80,000 or more had more costly and less efficient policing services than smaller cities, even in the presence of monitoring or auditing activities by citizens (Davis and Hayes (1993).)

The key economic problem facing a ministry responsible for the provision of a public good lies in the asymmetric information about costs between the producer of the good or service and the ministry.

Date: March 23, 2005

We would like to thank two anonymous referees for insightful comments that helped us to make significant improvements to the paper. We are grateful to Al Slivinski and Ig Horstmann for critique and valuable comments. The first author would like to thank Jim Davies for his support and encouragement and has benefited from discussions with Victor Aguirregabiria, Kathy Moscou, Jeffrey Smith, Ron Wintrobe, and Augustine Wong. 
This is true both if the producer is a profit maximizing private firm and if the producer is a government bureau. As a result, the ministry has to pay informational rents to each type of provider. If the provider is a profit maximizing firm, informational rents are minimized through the use of an auction mechanism, which introduces competition at the bidding stage (Osborne and Gaebler (1993).) McAfee and McMillan (1987) suggest that such competitive provision tends to involve first price sealed bid auctions.

Public sector bureaus have been characterized as Niskanen budget maximizing institutions (Niskanen (1975), Mueller (1997).) Bendor et al (1985) and Breton and Wintrobe (1975) suggest that monitoring and management compensation packages may lessen the informational rents of such Niskanen bureaus. Monitoring has been defined by Mookherje and Png (1992) as the commitment of resources towards the detection of an offense prior to receiving information about an occurrence. They term the commitment of resources after an offense has been reported as investigation. Auditing, as analyzed by Baron and Besanko (1984) in a principal-agent model with asymmetric information about a firm's realized costs, involves the expenditure of resources in order to detect misreporting after receiving a signal about costs.

This paper investigates the impact of budget size on the choice between auditing and competitive bidding in the provision of a publicly funded good. Auditing and competitive bidding raise several questions. First, what is the impact of the budget size on the funding source's choice between competitive bidding and auditing? Second, what cost distributions will result in the ministry having a non-trivial choice between competitive bidding and auditing the bureau for a given budgetary allocation? Third, what are the implications of the number of firms capable of providing the public sector good on the ministry's choice of competitive bidding or auditing?

In order to address these questions, the equilibrium outcomes of two mechanisms are compared: One is an auditing mechanism offered by the ministry, as principal, to a bureau, the agent. As in Baron and Besanko (1984) and Lacker and Weinberg (1989), the first-order approach to the principal-agent problem is used. In this approach, the global incentive compatibility constraint for the agent is replaced by a local incentive compatibility constraint, allowing the use of optimal control techniques. The second mechanism under investigation is an auction mechanism. Due to its greater analytical simplicity, a second price sealed bid auction is used to characterize the outcomes. The revenue equivalence theorem assures 
us that this auction will lead to the same (expected) utility for the ministry as the first-price sealed bid auction observed in practice. ${ }^{1}$

It is well known that an agent could be induced to truthfully report private information in the presence of infinite penalties for misreporting. The cultural norm that the "punishment fit the crime" however implies that there is a finite maximal penalty which the courts will uphold. This level is imposed exogenously. Auditing, thus, must occur with positive probability since even if the principal detects misreporting the penalty is bounded. Hence the auditing mechanism, just like the auction, is not capable of extracting all informational rents. Auctions, of course, are well known to be efficient mechanisms, i.e., to minimize the informational rents of the agent. Since in practice both auditing and auctioning are costly to implement, it is nevertheless not obvious which mechanism is better under what circumstances. The ministry thus faces a non-trivial choice between providing the publicly funded good either via competitive bidding or an audited bureau.

The main findings are as follows: Most generally, the optimal way to contract for the provision of the publicly funded good depends on the fixed costs of the mechanism, the number of potential bidders, and the distribution of costs. More specifically, we confirm that the informational rents of the provider increase faster with budget size under auditing than in the auction mechanism. This informational rent advantage of the auction mechanism can offset a fixed cost disadvantage relative to the audit mechanism. Hence, as the budget size increases, switching from auditing to the auction mechanism may occur at sufficiently high budget sizes even if auditing enjoys a fixed cost advantage over the auction mechanism. In the absence of this fixed cost advantage, the auction mechanism strictly dominates the audit mechanism for all budget sizes. An increase in the number of firms participating in the auction results in a decline in the budget level that induces switching from auditing to the auction, since the ministry's expected payoff from the auction is increasing in the number of bidders. We can also show that for small enough budget levels the ministry will choose to produce the good without auditing or conducting an auction, having some able provider do so at the highest possible cost, thereby avoiding any fixed costs associated with either mechanism. These results are consistent with the norm for many organizations that do not conduct

\footnotetext{
${ }^{1}$ See McAfee and McMillan (1987), or Milgrom (1987), among others. Jehle and Reny (2001) also provides an overview of auctions, the revenue equivalence theorem, and mechanism design.
} 
audits or auctions for sufficiently small procurement contracts or activities, as well as the examples at the beginning of the introduction. The resulting important role of the allocation of fixed costs in government is further discussed in the conclusion.

The paper is organized into five sections. In section 2 , the formal model is developed. Section 3 characterizes the equilibrium outcomes for the auditing and competitive bidding mechanisms. In section 4, the ministry's equilibrium choice is determined. Section 5 provides conclusions. All mathematical derivations and proofs are in a series of Appendices.

\section{The Model}

A ministry has an exogenously fixed program budget $R \in \Re_{+}$and cares only about the level of a publicly funded good, $x \in \Re_{+}$. The ministry does not possess the technology to produce the publicly funded good. Instead, the good is produced with a constant marginal cost technology by either a bureau or a firm. In either case, the actual level of marginal cost is private information. The ministry only knows that the marginal cost $c \in[\underline{c}, \bar{c}], \underline{c}>0$, is distributed according to a density $f(c)$.

The bureau is an extended Niskanen bureau that cares about its discretionary budget in excess of its production costs. Letting $B$ denote the budget transferred to the bureau, and $x$ the level of production, the bureau's preferences are represented by the utility function $v(B, x)=B-c x$. The bureau has a reservation level of utility, $\underline{v}$, which represents the payoff if its resources are re-deployed away from the provision of $x$. For simplicity, the reservation utility is assumed to be zero.

The firm is one of $N$ firms capable of producing the good. Firms are assumed to be (expected) profit maximizers with zero profits as their reservation utility. Each firm's actual marginal cost of production is determined by an independent, identically distributed draw from the cost distribution.

The ministry has three alternative institutional arrangements to obtain the publicly funded good, denoted by $a \in\{A, C, E\}$. It can design an auditing mechanism for the bureau (denoted by $A$ ); it can auction off the right to produce the good to one of the firms in a competitive bidding process, using a second-price sealed bid auction with a reserve price (denoted by $C$ ); or the ministry can choose to not design a mechanism at all using unsupervised production (denoted by $E$ ). In this last case, the ministry hands over all of the budget to a producer and demands the output level consistent with the highest 
possible cost realization. The resulting level of utility for the ministry, $R / \bar{c}$, constitutes a minimum utility level which can be achieved under either public or private provision.

2.1. Auditing. For auditing $(A)$, the ministry possesses an audit technology that generates a signal of true costs. This signal, denoted $\hat{c}$, is distributed on $[\underline{c}, \bar{c}]$. The distribution of the signal is conditional on the actual value of the bureau's cost realization, and has density $h(\hat{c} \mid c)$. The signal is assumed to be unbiased, that is $\int_{c}^{\bar{c}} \hat{c} h(\hat{c} \mid c) d \hat{c}=c$. It is also assumed that the conditional distribution satisfies the monotone likelihood ratio property: Let $H(\hat{c} \mid c)$ denote the (cumulative) distribution function, then it is assumed that $H(\hat{c} \mid c)<H\left(\hat{c} \mid c^{\prime}\right) \forall \hat{c}$ if $c>c^{\prime}$. Use of this audit technology costs resources. Let $q$ denote the probability of an audit occurring, then the associated audit cost is $m(q)$. The fixed cost of auditing, associated with maintaining the necessary infrastructure, is $m(0)=m_{0}$.

Assumption A1: Audit costs $m(q)$ satisfy: (i) $m(0)=m_{0}>0$; (ii) $m^{\prime}(q)>0, m^{\prime \prime}(q)>0$ for $q \in(0,1) ;\left(\right.$ iii) $m^{\prime}(0)=0$; and (iv) $\lim _{q \rightarrow 1} m(q)=\lim _{q \rightarrow 1} m^{\prime}(q)=\infty .^{2}$

If an audit is performed and generates a cost signal below the cost reported by the bureau, a penalty is imposed. This penalty is assumed to be exogenously determined by the courts and will be specified later. $^{3}$

Auditing is treated as a principal-agent problem and addressed in the usual mechanism design approach. The ministry is the principal and receives a report from the bureau, denoted by $w$. Based on this report, the principal chooses an audit contract consisting of a transfer from the ministry to the bureau, $t(w)$, the level of good provided by the bureau, $x(w)$, and an auditing intensity $q(w) \in[0,1]$. In the case where the audit returns a cost signal below reported cost, the bureau is penalized. Suppose that there exists an exogenous penalty function which furthermore is proportional to the mis-statement of costs, but which cannot reward the bureau; then, in the event that the bureau is audited, a penalty of $\max [0, p(w-\hat{c})]$ is assessed, where $\hat{c}$ is the cost signal, and $p$ is an exogenous parameter. ${ }^{4}$ The penalty is assumed to be paid into the consolidated revenue fund of the government and is not available to a

\footnotetext{
${ }^{2}$ A1 implies that the cost of monitoring, including the associated administrative costs, is convex. Hettich and Winer (1988) make a similar assumption about administrative costs and it is consistent with the empirical observations of Davis and Hayes (1993). Laffont and Tirole (1986) make a similar claim in relation to the disutility of effort in terms of money.

${ }^{3}$ Andreoni (1991), Bose (1995), and Dickens et al (1989) provide a comprehensive overview of penalties and their enforceability.

${ }^{4}$ Baron and Besanko (1984), in contrast, show that the optimal penalty function has the bang-bang feature for linear audit costs, for which an interior monitoring probability is a knife edge case.
} 
specific ministry. As in Baron and Besanko (1984) where the penalty is refunded to consumers and not to the regulator, there is no revision in the quantity of the good provided by the bureau after an audit.

The bureau's expected utility from stating a cost of $w$ when the true cost is $c$ is ${ }^{5}$

$$
\begin{aligned}
v(w ; c) & =(t(w)-c \cdot x(w))-q(w) \mathbf{E}_{\hat{c}}[\max [0, p(w-\hat{c})] \mid c] \\
& =(t(w)-c \cdot x(w))-q(w) p \int_{\underline{c}}^{w}(w-\hat{c}) h(\hat{c} \mid c) \mathrm{d} \hat{c} \\
& =t(w)-c \cdot x(w)-q(w) p \int_{\underline{c}}^{w} H(\hat{c} \mid c) \mathrm{d} \hat{c} .
\end{aligned}
$$

It is important to note that both $v(w ; c)$ and $\partial v(w ; c) / \partial w$ are continuous at $w=c$.

By the revelation principle, attention can be restricted to truth-telling mechanisms. The participation constraint for the bureau is given by

$$
v(c, c)=t(c)-c \cdot x(c)-q(c) p \int_{\underline{c}}^{c} H(\hat{c} \mid c) \mathrm{d} \hat{c} \geq 0 .
$$

Global incentive compatibility requires that

$$
v(c ; c) \geq v(w ; c) \quad \forall w, c \in[\underline{c}, \bar{c}] .
$$

We employ a local incentive compatibility approach, as shown in Appendix (A). This restricts the derivative of the bureau's value function $v(c)$, denoted by $\dot{v}(c)$, and is given by

$$
\dot{v}(c)=-x(c)-q(c) p \int_{\underline{c}}^{c} \frac{\partial H(\hat{c} \mid c)}{\partial c} \mathrm{~d} \hat{c} .
$$

The ministry's problem then is to maximize its expected payoff by choosing a contract $(t(w), x(w), q(w))$ to offer to the bureau for any reported cost $w$. It faces the budget constraint $R-m(q(w))-t(w) \geq 0$, as well as the participation constraint (2) and the incentive compatibility constraint (4). Optimal control theory is used to solve this problem. Specifically, the bureau's utility $v(\cdot)$ is considered to be the state variable and the control variables are $t(w), x(w)$, and $q(w)$. Truth-telling together with (2) implies:

$$
t(c)=v(c)+c \cdot x(c)+q(c) p \int_{\underline{c}}^{c} H(\hat{c} \mid c) \mathrm{d} \hat{c},
$$

\footnotetext{
${ }^{5}$ Note that $\int_{\underline{c}}^{w} w h(\hat{c} \mid c) \mathrm{d} \hat{c}=w H(w \mid c)$, while integration by parts yields $\int_{\underline{c}}^{w} \hat{c} h(\hat{c} \mid c) \mathrm{d} \hat{c}=w H(w \mid c)-\int_{\underline{c}}^{w} H(\hat{c} \mid c) \mathrm{d} \hat{c}$.
} 
so that $t(w)$ can be eliminated as a control. The ministry's problem then is: ${ }^{6}$

$$
\begin{aligned}
& \max _{q(\cdot), x(\cdot)}\left\{\int_{\underline{c}}^{\bar{c}} x(s) f(s) \mathrm{d} s\right\} \\
& \text { s.t. (i) } \dot{v}(c)=-x(c)-q(c) p \int_{\underline{c}}^{c} \frac{\partial H(\hat{c} \mid c)}{\partial c} \mathrm{~d} \hat{c} \\
& \text { (ii) } R-m(q(c))-v(c)-x(c) c-q(c) p \int_{\underline{c}}^{c} H(\hat{c} \mid c) \mathrm{d} \hat{c} \geq 0 \\
& \text { (iii) } v(c) \geq 0 \\
& \text { (iv) } v(\bar{c})=0
\end{aligned}
$$

This problem is analysed fully in Appendix (B). A priori there are two cases of interest: first, when both the transition equation (i) and the budget constraint (ii) are binding, and, second, the case when the budget constraint (ii) is slack while positive output is produced, indicating that the ministry funds some production but has unused budget room. The latter cannot be part of the solution however. The intuition behind this is as follows: Since the ministry does not care about money but only output, the free budget does not add to utility. It could be spent instead on an increased audit probability, which reduces the slope of the value function, relaxing the incentive compatibility constraint, and thus allowing for higher output. Hence the ministry would increase the audit probability (and output), ultimately exhausting the budget. It follows that the solution to (6) will involve both the transition equation and the budget constraint binding at positive output levels.

Just as in an auction with a reserve price, the optimal contract may involve a region of cost reports for which the ministry does not fund the production of output by the bureau. Denote this cutoff value above which no production occurs by $\hat{r}$. It is determined by the distribution of costs according to $\hat{r} f(\hat{r})-F(\hat{r})=0$ (see Appendix B.) ${ }^{7}$ As in an auction with a reserve price, it may be better for the ministry (in expected utility terms) not to receive output for some (high) realizations of cost and in turn to receive more output if the cost realization is low. The exclusion of the high cost types relaxes the incentive compatibility constraint for the lower cost types and helps to reduce their informational rents. In the optimal contract the ministry audits with $q(c ; p)>0$ for cost reports in the interval $c \in[\underline{c}, \hat{r})$ where

\footnotetext{
${ }^{6}$ See Kamien and Schwartz (1981) for an outline of the techniques used to solve such programming problems.

${ }^{7}$ As an example, the p.d.f. $f(c)=2 / c^{3}, 1<c<\infty$ from Hoag and Craig (1978, p. 32) can be shown to result in $\hat{r}=3^{1 / 2}$ and $F(\hat{r}) / \hat{r}=10 / 27$ for $\hat{r}=\{r: f(r) r-F(r)=0\}$.
} 
it transfers funds and demands output. The minstry does not audit $(q(c ; p)=0)$ for cost reports $c \geq \hat{r}$, and does not transfer funds nor demand output.

2.1.1. Ministry Payoff. The ministry's expected (over cost realizations) payoff from the audit mechanism is denoted by $E_{c}[u(c, R: A)]$. In what follows, this payoff schedule will be characterized.

By definition, $\mathrm{E}_{c}[u(c, R: A)]=\int_{\underline{c}}^{\bar{c}} x(s) f(s) \mathrm{d} s$. Let $\hat{r} \leq \bar{c}$ denote the highest cost for which a positive output is required as per the discussion above. Using (6(ii)) to isolate $x(\cdot)$, the ministry's expected payoff schedule can be rewritten as:

$$
\int_{\underline{c}}^{\hat{r}} x(s) f(s) \mathrm{d} s=\int_{\underline{c}}^{r}\left(-\dot{v}(s)-q(s) p \int_{\underline{c}}^{s} \frac{\partial H(\hat{c} \mid s)}{\partial s} \mathrm{~d} \hat{c}\right) f(s) \mathrm{d} s .
$$

Using the solution to the optimal control problem (6), it is shown in Appendix (C) that the ministry's expected payoff schedule can be expressed as:

$$
\mathrm{E}_{c}[u(c, R: A)]=\int_{\underline{c}}^{\hat{r}}\left(-K(R)+\chi(s ; p)+\frac{R-m_{0}}{s}-\frac{1}{s} \int_{\underline{c}}^{s} \zeta(t ; p) \mathrm{d} t-\frac{q(s) p}{s} \int_{\underline{c}}^{s} H(\hat{c} \mid s) \mathrm{d} \hat{c}\right) f(s) \mathrm{d} s,
$$

where the functions $\chi(c ; p)$ and $\zeta(t ; p)$ are as defined in equations $(\mathrm{C} 3)$ and $(\mathrm{B} 12)$, respectively. $K(R)$ is a constant of integration which depends on the budget level $R$. Appendix (C) also shows that

$$
\frac{\partial \mathrm{E}_{c}[u(c, R: A)]}{\partial R}=\frac{1}{\hat{r}} F(\hat{r})>0 .
$$

Hence the ministry's expected payoff is linear and increasing in the budget level, and we have an expression for the slope which we can be used to compare provision through auditing versus competitive bidding.

2.2. Competitive bidding. Under competitive bidding $(C)$, the ministry conducts a second-price sealed bid auction with a reserve price. The auction incurs a fixed cost $b_{F} \geq 0$. This fixed cost captures the costs of the necessary infrastructure associated with operating the auction, which may include the cost of hiring staff for drafting the bid, addressing questions, and evaluating the responses, as well as advertisement costs and the costs of other regulatory requirements implicit in public sector bidding activities. The free budget available for goods provision then is $\left(R-b_{F}\right)$. In the auction, the ministry accepts sealed bids on per unit cost from the $N$ firms that are capable of producing the good. If all bids exceed the reserve price no production will occur and no budget is transfered. Otherwise, the lowest cost bid wins the auction and in return receives the available budget. The winning bidder is required to produce output at a level 
corresponding to the lower of the unit cost of the second lowest bid or the reserve price. The output level thus will be $x=\left(R-b_{F}\right) / c$ units, where $c$ denotes that unit cost.

The strategies for the $N$ firms are their bids $w\left(c_{n}\right)$. As usual, the firms' weakly dominant strategy in a second-price sealed bid auction is to bid truthfully, that is $w\left(c_{i}\right)=c_{i}$. This is due to the fact that a firm's payoff, conditional on winning the auction, is independent of its bid. Let $c=\left(c_{1}, c_{2}, \ldots, c_{N}\right)$ denote the $N$-vector of submitted bids. The $i^{t h}$ order statistic is denoted by $c_{(i)}$, such that $c_{(1)} \leq c_{(2)} \leq \cdots \leq c_{(N)}$. $G_{(i)}$ denotes the probability distribution of this $i^{\text {th }}$ order statistic of true costs, with the associated density $g_{(i)}$. Since costs are distributed iid according to $F(\cdot)$ with density $f(\cdot)$, we know that ${ }^{8}$

$$
\begin{aligned}
G_{(i)}(c) & =\sum_{u=i}^{N}\left(\begin{array}{l}
N \\
u
\end{array}\right) F(c)^{u}(1-F(c))^{N-u} \\
g_{(i)}(c) & =\frac{N !}{(i-1) !(N-i) !} f(c) F(c)^{i-1}(1-F(c))^{N-i} .
\end{aligned}
$$

Let $r$ denote the reservation level of cost, above which no production occurs. ${ }^{9}$ There are three relevant events in computing the ministry's expected utility from the auction: (1) If $c_{(1)}>r$ then no bid is below the reserve level and the ministry obtains a zero payoff from the auction since no private firm production is funded. This event occurs with probability $\left(1-G_{(1)}(r)\right)$. (2) If $c_{(2)}<r$ then at least two bids are below the reserve level. In this case the level of output will correspond to the second lowest of these $\operatorname{costs} c_{(2)}$, while production and all of the budget net of fixed costs is awarded to the lowest bidder. Hence the output level will be $\left(R-b_{F}\right) / c_{(2)}$. Of course, $c_{(2)}$ is a random variable, and the expectation needs to be taken over the interval $(\underline{c}, r)$ using the density function $g_{(2)}(c)$. (3) If $c_{(1)}<r$ but $c_{(2)}>r$ then the lowest bid will win at the reserve level of cost. Hence production occurs with an output level corresponding to a production cost of $r$. The associated output level is $\left(R-b_{F}\right) / r$. Production is by the lowest cost firm, which receives all of the budget net of fixed costs. The probability of this event is $\left(1-\left[1-G_{(1)}(r)\right]-G_{(2)}(r)\right)=G_{(1)}(r)-G_{(2)}(r)$, since with probability $1-G_{(1)}(r)$ event $(1)$ occurs, with probability $G_{(2)}(r)$ event $(2)$ occurs, and the three events are mutually exclusive and exhaustive. ${ }^{10}$

\footnotetext{
${ }^{8}$ See, for example, David, H.A., 1981, Order Statistics, 2nd edition, John Wiley and Sons Inc, New York.

${ }^{9}$ Note that its value is, a priori, different from the value of $\hat{r}$ in the previous section.

${ }^{10}$ It can be shown that $\left.G_{(1)}(r)\right)-G_{(2)}(r)=\left(\begin{array}{c}N \\ 1\end{array}\right) F(r)[1-F(r)]^{N-1}$.
} 
We can now state the ministry's expected utility from the auction, denoted $E_{c}[u(c, R, N: C)]$, which it will maximize by choosing an optimal $r$ :

$$
\left.E_{c}[u(c, R, N: C)]=\int_{\underline{c}}^{r} \frac{R-b_{F}}{c} g_{(2)}(c) \mathrm{d} c+\left[G_{(1)}(r)\right)-G_{(2)}(r)\right] \frac{R-b_{F}}{r} .
$$

The optimal reserve price $r^{*}$ for the ministry is derived in Appendix (D), where it is shown that it satisfies:

$$
r^{*}=\frac{F\left(r^{*}\right)}{f\left(r^{*}\right)} \quad \text { if } \quad f^{\prime}\left(r^{*}\right)\left(1-F\left(r^{*}\right)\right) \leq 2(N-2) f\left(r^{*}\right)^{2} .
$$

Equation (13) is related to the usual monotonic hazard rate condition. In particular, the standard assumption that $v-(1-F(v)) / f(v)$ is nondecreasing implies that $f^{\prime}(r)(1-F(r)) \leq 2 f(r)^{2}$, which clearly is sufficient to allow (13) to hold for all $N>3$. Hence, a competitive sector of more than three firms is a sufficient condition for $r^{*}$ to define an equilibrium reservation level of cost for the ministry. ${ }^{11}$ It is interesting to observe that the cultural or institutional norm in many public institutions is to require a minimum of three bids or quotes.

2.2.1. Ministry payoff. For $r^{*}$ satisfying (13), the expected profits of the ministry are

$$
\begin{aligned}
& E_{c}[u(c, R, N: C)]=\left\{R-b_{F}\right\}\left\{\frac{1}{\underline{c}}-\frac{1}{r^{*}}\left(1-F\left(r^{*}\right)\right)^{N}\right. \\
& \left.-\int_{\underline{c}}^{r^{*}} \frac{(1-F(c))^{N}}{c^{2}} \mathrm{~d} c-\int_{\underline{c}}^{r^{*}} \frac{N F(c)(1-F(c))^{N-1}}{c^{2}} \mathrm{~d} c\right\} .
\end{aligned}
$$

$\mathrm{E}_{c}\left[u(c, R, N: C)\right.$ approaches $\left\{R-b_{F}\right\} / \underline{c}$ as $N$ approaches infinity. For $R \leq b_{F}$, the ministry can choose to do nothing and $E_{c}[u(c, R, N: C)=0$ in that case. The expected payoff is strictly positive otherwise. Clearly, it is linear and increasing in $R$, since the term in the large brackets above is independent of $R$.

\section{Characterization of institutional Choice}

The institutional choice on the part of the ministry involves the ministry choosing to have the good produced in the public sector through auditing or in the private sector through competitive bidding, provided that one of them produces a higher expected utility than $R / \bar{c}$. This lower bound derives from the fact that the ministry can have any firm or bureau provide the good at this cost level without supervision. The ministry chooses competitive bidding if $E_{c}[u(c, R, N: C)] \geq \max \left\{E_{c}[u(c, R: A)], \frac{R}{\bar{c}}\right\}$,

\footnotetext{
${ }^{11}$ Note that the exclusion level of costs in the auditing framework, following equation (B.13) in Appendix B2, is defined by the same condition as here for the auction mechanism. It follows that auctions and auditing both exclude the same set of cost realizations.
} 
and audited provision by a bureau if $E_{c}[u(c, R: A)] \geq \max \left\{E_{c}[u(c, R, N: C)], \frac{R}{\bar{c}}\right\}$. If neither inequality holds the provision is by either method, since an unsupervised provider is engaged.

Of particular interest is the question how the institutional choice depends on the budget size and how it varies with it. As has already been noted, both institutions' expected payoffs are linear in the budget, $R$. It was shown earlier that $\partial \mathrm{E}_{c}[u(c, R: A)] / \partial R=F(\hat{r}) / \hat{r}$ in the case of auditing, while it follows from (14) that for an auction

$$
\frac{\partial E_{c}[u(\cdot)]}{\partial R}=\frac{1}{\underline{c}}-\frac{\left(1-F\left(r^{*}\right)\right)^{N}}{r^{*}}-\int_{\underline{c}}^{r^{*}} \frac{(1-F(c))^{N}}{c^{2}} \mathrm{~d} c-\int_{\underline{c}}^{r^{*}} \frac{N F(c)(1-F(c))^{N-1}}{c^{2}} \mathrm{~d} c .
$$

Clearly, for the auction $\lim _{N->\infty} \partial \mathrm{E}_{c}[u(\cdot)] / \partial R=1 / \underline{c}$. Therefore the expected payoff is increasing more rapidly under competitive bidding than under auditing if there is a very large number of firms. On the other hand, for $N=1, \partial \mathrm{E}_{c}[u(c, R, 1: C)] / \partial R=F\left(r^{*}\right) / r^{*}$. Note that $r^{*}=\hat{r}$, since both values are defined by the same equation. Of course, $N=1$ is not a valid number of firms in an auction. However, since $\partial \mathrm{E}_{c}[u(c, R, N: C)] / \partial R>\partial \mathrm{E}_{c}[u(c, R, N-1: C)] / \partial R$ for all $N$ (see Appendix $\left.(\mathrm{F})\right)$ it follows that for all valid values of $N$ (i.e., $N \geq 3$ ) the expected payoff from the auction is increasing more rapidly than that of the auditing mechanism.

What is the intuition behind this result? It derives from the well known fact that auctions are optimal mechanisms, that is, extract the maximal amount of informational rents from the informed agent. The auditing scheme chosen is not able to achieve the same degree of rent extraction. It follows that the competitive mechanism yields a higher increment in expected utility to the ministry than the audit mechanism for any given increment in budget size.

More explicitly, since the providers' reservation utility has been normalized to zero, interim informational rents (that is, the rents once the provider knows true cost) can be defined as the expected profits of a provider from participating in the mechanism. ${ }^{12}$ For the auction mechanism the profits for a firm with cost realization $c_{(1)}$ are

$$
\min \left\{\left(\left(R-b_{F}\right) \frac{c_{(2)}-c_{(1)}}{c_{(2)}}\right),\left(R-b_{F}\right) \frac{r^{*}-c_{(1)}}{r^{*}}\right\}
$$

since the required output corresponds to either the second lowest cost or the reserve, whichever is lower. For the audit mechanism the bureau's utility is given by $v(c)$ defined in equation (B.16) in Appendix

\footnotetext{
${ }^{12}$ Note that under full information each provider would be held to the reservation level of utility for all cost realizations.
} 
B. While these expressions do not lend themselves to easy comparison, it can be noted that for both the auction and the audit mechanism the informational rent of a provider at the (identical) reservation cost levels $\left(\hat{r}, r^{*}\right)$ is zero. For all lower cost realizations informational rents accrue. They are higher for the audited bureau compared to the winning bidder in an auction. Furthermore, as the budget size is increased, informational rents also increase. For the auction the rate of increase is

$$
\min \left\{1-\frac{c_{(1)}}{c_{(2)}}, 1-\frac{c_{(1)}}{r^{*}}\right\} .
$$

For the bureau, the rate of increase in informational rents is

$$
\frac{\partial v(c)}{\partial R}=c \frac{\partial K(R)}{\partial R}-c \int_{\underline{c}}^{c} \frac{1}{s^{2}} \mathrm{~d} s=1-\frac{c}{\hat{r}} .
$$

Clearly, then, the bureau obtains a larger increase in informational rents unless the auction happens to have the second lowest cost realization at or above the reservation level — an event that occurs with a probability strictly less than one.

Informational rent considerations alone would thus suggest that competitive provision is always superior to audited provision. However, the ministry's expected payoff not only depends on the informational rents given up, but also on the costs associated with operating each of the mechanisms. These costs have the effect of reducing the budget available for actual goods provision. Since the auction is a more efficient mechanism, it will dominate the audited provision anytime the actual budget available for goods production is the same for both mechanisms. Of course, if auctions are not only more efficient in terms of informational rents, but also cost less to run in terms of their fixed costs $\left(b_{F}\right)$ compared to the (expected) auditing fixed costs, then the auction will definitely yield a higher ministry utility. ${ }^{13}$

An example of this case is depicted in Figure 1, where ministry expected utility is plotted against budget size for unsupervised provision, competitive provision, and audited provision. As the figure demonstrates, the ministry will choose competitive bidding for all sufficiently high budget levels $R>R_{0}(N)$, where: $R_{0}(N)=\left\{R: E_{c}[u(c, R, N: C)]-R / \bar{c}=0\right\}$. If $R \leq R_{0}(N)$, then the ministry chooses to produce the good without oversight, earning a payoff $R / \bar{c}$. Since the expected utility from the competitive mechanism depends positively on the market depth $N$, it is clear that $R_{0}(N)$ will be lower the larger is $N$. Note

\footnotetext{
${ }^{13}$ The expected fixed cost of auditing is denoted by $E[M+P]$ for simplicity. It consists of the expected costs of performing the audit, plus the expected value of the funds transferred to the bureau in order to cover the bureau's expected penalties.
} 


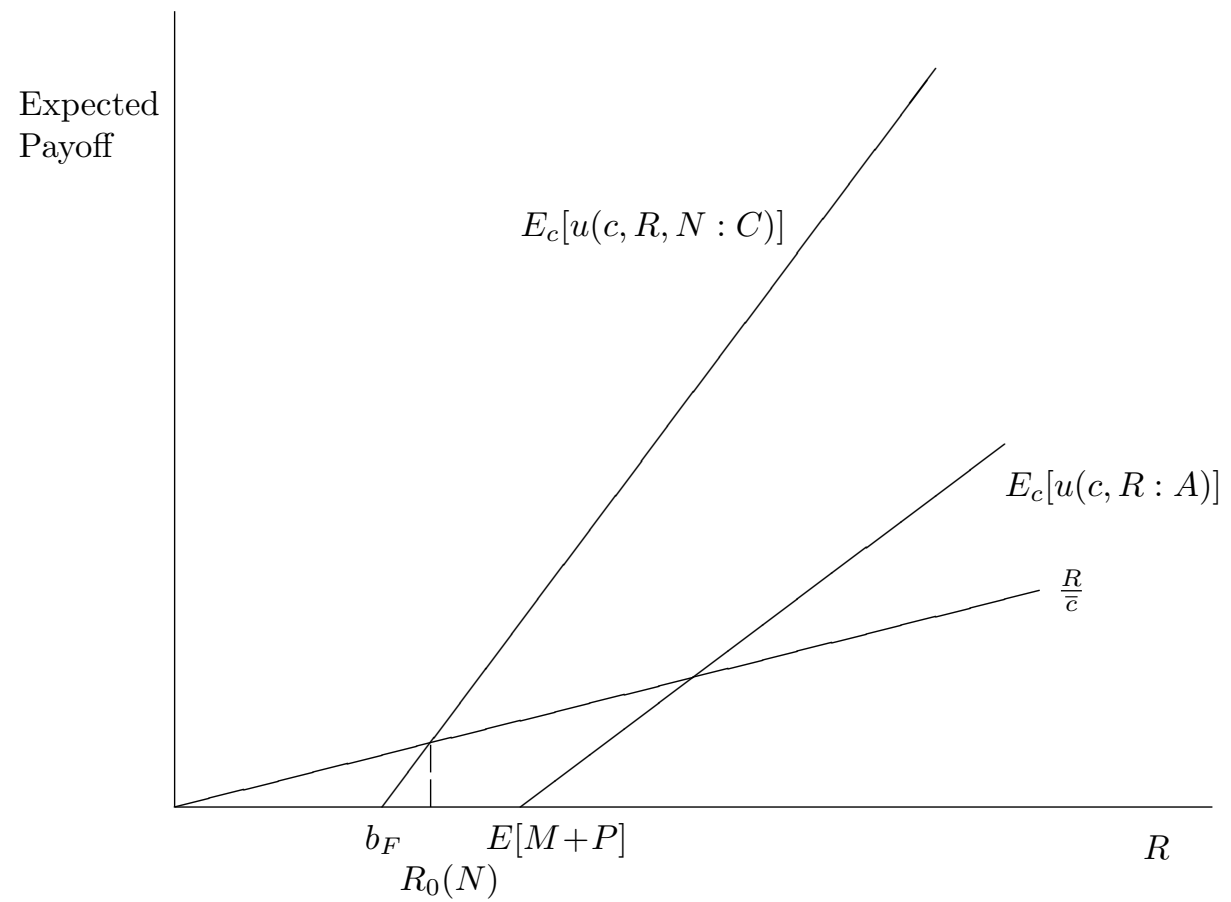

Figure 1. Expected payoffs if $E[M+P]>b_{F}$

also that "mandatory competitive provision" policy would only be optimal if auction costs are not higher than any costs associated with completely unsupervised provision (here, zero.)

If, however, the costs associated with running an auction $\left(b_{F}\right)$ exceed the (expected) costs associated with auditing, then the audit allows a larger budget to be allocated to goods production. Even though the audit is not as efficient in transforming this budget into ministry utility, it may yield a higher ministry utility due to the larger budget available for production.

Figure 2 shows an example of this situation. There now exist two critical budget sizes at which provision choice may switch. At the first, $R_{1}$, the audited bureau becomes better than unsupervised high cost provision, at the second, $R_{2}(N)$, competitive provision starts to outperform the audited bureau. Here $R_{1}=\left\{R:\left[E_{c}[u(c, R,: A)-R / \bar{c}]=0\right\}\right.$ and $R_{2}(N)=\left\{R: E_{c}[u(c, R, N: C)]-E_{c}[u(c, R,: A)]=0\right\}$. The ministry chooses competitive bidding if $R>R_{2}(N)>R_{1}$ or if $R>R_{0}(N), R_{2}(N)<R_{1}$. If $R_{2}(N) \geq R \geq R_{1}$, the ministry chooses auditing, and the ministry conducts no supervision (or oversight) if $R \leq R_{1}$. As in the previous case, since the marginal expected payoff of competitive provision depends positively on the number of firms participating in the auction $R_{2}(N)$ is decreasing in $N$.

The following proposition summarizes these results. 


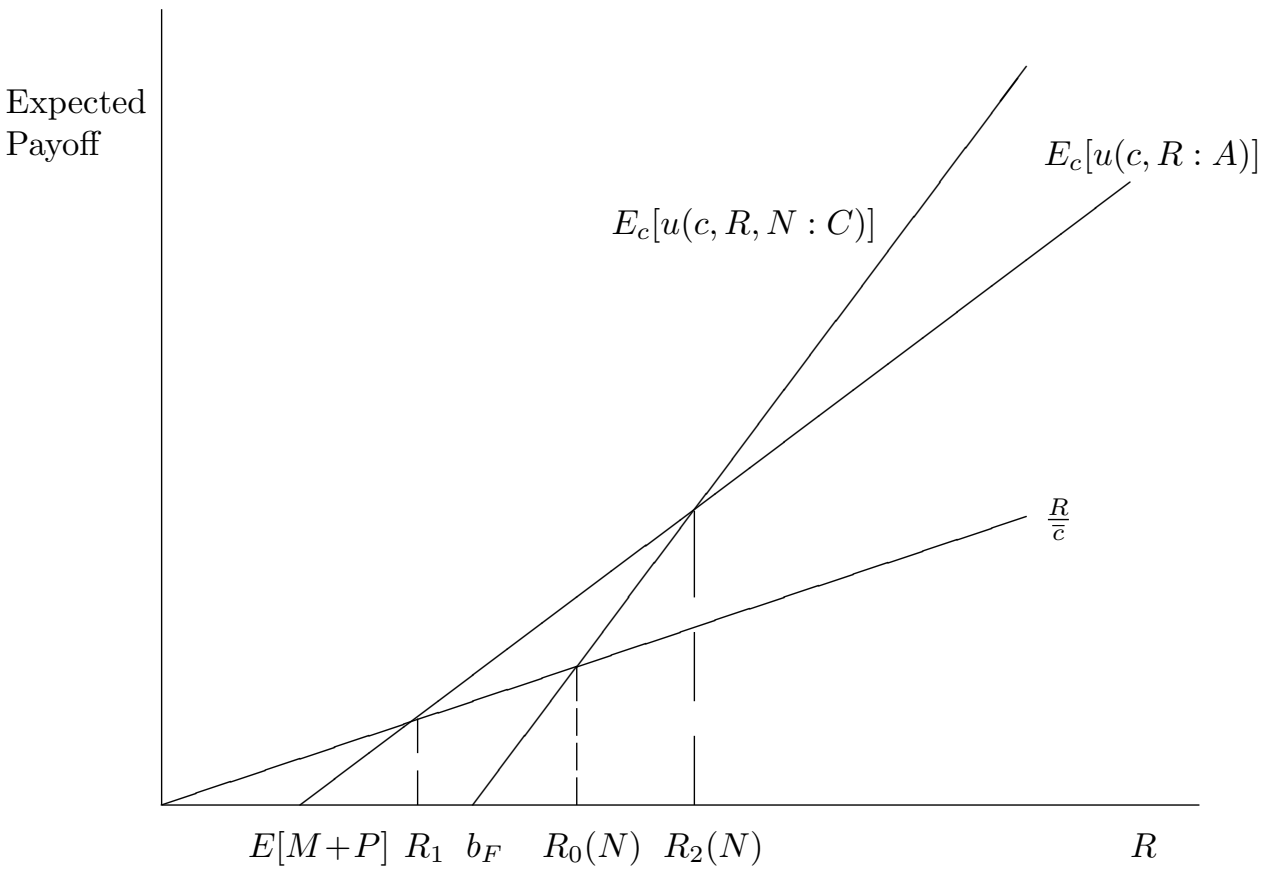

Figure 2. Expected payoffs if $E[M+P]<b_{F}$

Proposition 1. If auctions have lower fixed costs than auditing, the ministry will choose a competitive bidding process for all sufficiently high budget levels. If, however, auditing has sufficiently lower (expected) costs, the ministry may first switch from high cost provision to an audited bureau as budget size increases, and only for even higher budget levels will competitive provision become optimal. In either case, the budget level at which competitive provision becomes optimal is lower, the larger the competitive sector.

This proposition predicts that the institutional arrangement in the public sector is dependent on the size of the ministry's budget, the size of the competitive market, and the relative sizes of the fixed costs of auditing and auctioning. This latter point is of particular policy relevance, since the allocation of fixed costs of supervision or competitive procurement is endogenous in multi-product government settings. As is further discussed below, the optimality of competitive provision can therefore be affected by administrative rules concerning cost allocation.

\section{Discussion}

The paper analyses a ministry's choice in the provision of a public sector good in the presence of asymmetric information. The ministry copes with asymmetric information about provision costs by 
either auditing a Niskanen budget maximizing bureau, or by contracting with private firms. In the case of auditing, the penalty which can be imposed is exogenously limited, limiting the minstry's abilty to extract informational rents from the bureau. In the contracting case, a second price sealed bid auction is used in order to minimize informational rents. The paper extends the Niskanen model of government bureaucracy, and endogenizes the choice between auditing and competitive bidding in the public sector.

We show that at very low budget levels it may be optimal not to invest any resources towards the reduction of informational rents, but to provide the good without any supervision. This result is consistent with the common practices of many public institutions that permit the issuance of procurement contracts to a single firm without tender when these contracts are sufficiently small. It is noteworthy that the implementation of government regulations to require the use of auctions independent of the budget level may therefore not imporove welfare.

For higher budget levels the optimal choice involves an attempt to limit informational rents. The approach that is best will depend on the costs associated with each and the budget size. An auction is the optimal choice independent of the size of the budget if the fixed costs for competitive bidding are low relative to those expected for auditing. If, however, the fixed costs for competitive bidding are high relative to those for auditing, the optimal choice can depend on the budget level. In that case the institutional arrangement can involve switching from public sector to private sector production as budget size increases. This is driven by the fact that auctions are the optimal way to limit informational rents, and so will ultimately outperform any other mechanism (e.g., auditing and unsupervised production) even if it has an initial cost advantage.

Both auctions and audited provision may involve a reservation level of cost, above which the ministry will not fund the provision of the good. The optimal level of this reservation cost depends on the distribution of costs, as is usual for auctions and shown here for auditing. Indeed, it is the same for both procurement choices. As is common practice for public sector procurement, it is found that the auction mechanism requires more than three firms to participate.

The size of government is reportedly positively correlated to overall economic activity as measured in terms of gross domestic product per capita (Alesina and Wacziary, 1998). Holsey and Borcherding (1997) 
also suggest that government budget rises faster than income in the short run while its long-run income elasticity is unity (i.e., a restating of Wayne's Law). While Wayne's Law is not supported by all empirical studies, the size of government is generally found to be positively correlated to per capita income and the income of the median voter. ${ }^{14}$ It follows that the results predict that there will be growing reliance on private provision of public sector goods during periods of economic boom and expanding government budgets even when fixed costs favor auditing over auctions. Also, developed countries tend to have larger government budgets in absolute and as a percentage of GDP than developing nations. Again, if fixed costs, including the availability of technical ability, favor auditing over auctions, then it can be anticipated that the private sector will be more appropriately involved in the provision of public sector goods in developed countries as compared to developing nations. In general, the implementation of institutional arrangements in government without regard for the fixed costs of maintaining these structures or the size of the government budgetary resources may not be welfare improving.

This study predicts that the government copes with information asymmetries by aiming to minimize informational rents. The optimal institutional arrangement of government is found to vary depending on the costs of each arrangement. However, in a government environment with multiple products, the allocation of fixed costs between programs is endogenous. Since it also is a significant determinant of the choice between public or private sector provision, this study suggests that the administrative rules associated with the allocation of these costs are important in predicting and determining the institutional arrangements of government. Bureaucrats can influence these fixed costs through the allocation methodology, and may therefore be instrumental in determining the fixed cost of conducting public sector auctions or public sector supervision. Hence, the institutional rules surrounding the allocation of these costs are an important determinant of the institutional arrangements established for the provision of public sector goods.

\footnotetext{
${ }^{14}$ Alesina and Wacziary (1998) report that total government expenditures, including transfers and interest payments, as a percentage of GDP is positively related to per capita income based on a cross-section analysis of over 90 countries. This relationship also holds for public expenditures on defense, education and public investment when population has been taken into account.
} 


\section{APPENDIX}

\section{A. Local Incentive Compatibility in Auditing:}

The global incentive compatibility constraint (3) implies an infinite number of constraints. Rogerson (1985) and Jewitt (1988) outline the difficulties involved in using this type of constraint. The use of a local incentive compatibility constraint is suggested as an alternative to the global approach, and is also used by Baron and Besanko (1984), for example. The local incentive compatibility constraint approach uses the fact that the optimality of truth telling involves locally maximizing the bureau's utility or payoff. The first order condition for the bureau's optimal choice of reported cost is $\frac{\partial v(w ; c)}{\partial w}=0$. Together with truth telling $(w=c)$, we therefore require

$$
\left.\frac{\partial v(w ; c)}{\partial w}\right|_{w=c}=t^{\prime}(c)-c \cdot x^{\prime}(c)-q^{\prime}(c) p \int_{\underline{c}}^{c} H(\hat{c} \mid c) \mathrm{d} \hat{c}-q(c) p H(c \mid c)=0 .
$$

The second order condition is

$$
\left.\frac{\partial^{2} v(w ; c)}{\partial w \partial w}\right|_{w=c}=t^{\prime \prime}(c)-c \cdot x^{\prime \prime}(c)-q^{\prime \prime}(c) p \int_{\underline{c}}^{c} H(\hat{c} \mid c) \mathrm{d} \hat{c}-2 q^{\prime}(c) p H(\hat{c} \mid c)-q(c) p h(c \mid c) \leq 0 .
$$

Let $v(c)=v(c, c)$ denote the resulting value function for the bureau under truth telling. By the envelope theorem

$$
\dot{v}(c)=-x(c)-q(c) p \int_{\underline{c}}^{c} \frac{\partial H(\hat{c} \mid c)}{\partial c} \mathrm{~d} \hat{c} .
$$

This restriction will be imposed on the equilibrium value function and takes the place of (3).

\section{B. The Optimal Control Problem:}

The ministry's problem is:

$$
\begin{aligned}
& \max _{q(\cdot), x(\cdot)}\left\{\int_{\underline{c}}^{\bar{c}} x(s) f(s) \mathrm{d} s\right\} \\
& \text { s.t. }(\text { i }) \quad \dot{v}(c)=-x(c)-q(c) p \int_{\underline{c}}^{c} \frac{\partial H(\hat{c} \mid c)}{\partial c} \mathrm{~d} \hat{c} \\
& \text { (ii) } R-m(q(c))-v(c)-x(c) c-q(c) p \int_{\underline{c}}^{c} H(\hat{c} \mid c) \mathrm{d} \hat{c} \geq 0 \\
& \text { (iii) } v(c) \geq 0 \\
& \text { (iv) } v(\bar{c})=0
\end{aligned}
$$


Let $\lambda(c)$ denote the (differentiable) multiplier on the transition equation (i) and let $\mu(c)$ denote the (differentiable) multiplier on (ii), which has to satisfy:

$$
\mu(c) \geq 0 ; \quad \mu(c)\left(R-m(q(c))-v(c)-x(c) c-q(c) p \int_{\underline{c}}^{c} H(\hat{c} \mid c) \mathrm{d} \hat{c}\right)=0 .
$$

The first order necessary conditions for the problem in (B.1) are:

$$
\begin{aligned}
& R-m(q(c))-v(c)-x(c) c-q(c) p \int_{\underline{c}}^{c} H(\hat{c} \mid c) \mathrm{d} \hat{c} \geq 0 \perp \mu(c) \geq 0 \\
& \dot{v}(c)=-x(c)-q(c) p \int_{\underline{c}}^{c} \frac{\partial H(\hat{c} \mid c)}{\partial c} \mathrm{~d} \hat{c} \\
& v(\bar{c})=0 \\
& -\lambda^{\prime}(c)=-\mu(c) \\
& \lambda(\underline{c})=0 \\
& f(c)-\lambda(c)-c \mu(c)=0 \\
& -\lambda(c) p \int_{\underline{c}}^{c} \frac{\partial H(\hat{c} \mid c)}{\partial c} \mathrm{~d} \hat{c}-\mu(c) p \int_{\underline{c}}^{c} H(\hat{c} \mid c) \mathrm{d} \hat{c}-\mu(c) m^{\prime}(q(c))=0
\end{aligned}
$$

The Kuhn-Tucker constraint qualifications will hold as long as (B.1)(ii) is concave.

\section{Constraints:}

Transition equation and budget constraint binding. The transition equation and the budget constraint are binding if $\lambda \neq 0$ and $\mu \neq 0$. Equations (B.6) and (B.8) then imply the linear first order differential equation

$$
\lambda^{\prime}(c)+\lambda(c) \frac{1}{c}=\frac{f(c)}{c}
$$

and hence

$$
\lambda(c)=\frac{1}{c}(F(c)+k)
$$

for some constant $k$. Since $\lambda(\underline{c})=0$ by (B.7), it follows that $k=0$, and so:

$$
\lambda(c)=F(c) / c \quad \text { if } \quad \mu(c)>0 .
$$

Knowing $\lambda(c)$, equation (B.9) gives rise to:

$$
m^{\prime}(q(c ; p))=-p\left[\frac{c F(c)}{(f(c) c-F(c))} \int_{\underline{c}}^{c} \frac{\partial H(\hat{c} \mid c)}{\partial c} \mathrm{~d} \hat{c}-\int_{\underline{c}}^{c} H(\hat{c} \mid c) \mathrm{d} \hat{c}\right] .
$$

Denote the RHS of (B.13) by $\zeta(c ; p)$. Since $\partial H(\cdot, c) / \partial c<0$, the first term in the square brackets is negative for low $c$. The second term is, of course, positive, so that the square brackets are guaranteed 
to be negative. At $c=\underline{c}$, the square bracket is zero, so that $q(\underline{c} ; p)=0$. The audit intensity, $q(c, p)$, is increasing in the penalty $p$. Clearly, in the absence of a penalty (i.e., $p=0$ ) the ministry has no incentive to audit the bureau as auditing would provide no deterrent to misreporting. For $p>0$, an audit does serve as a deterrent and so $q(c ; p)>0$ as characterized by equation (B.13).

Suppose there exists an $\hat{r} \in[\underline{c}, \bar{c}]$ such that $\hat{r} f(\hat{r})-F(\hat{r})=0$. As $c \rightarrow \hat{r}$ from below the first term in the square brackets approaches negative infinity. The implication is that marginal monitoring cost will also have to approach infinity. Since total monitoring cost then will also become large (by A1) this is inconsistent with (B.1(ii)). It follows that a corner solution must occur at some point prior to (at a cost below) $\hat{r}$. In that case, auditing occurs with $q(c ; p)>0$ for the set of $c \in[\underline{c}, \hat{r})$ and $q(c ; p)=0$ for $c>\hat{r}$ since a reported cost above the reservation price $(\hat{r})$ results in the ministry not funding the production of the good.

Given $\lambda(\underline{c})=0$, it follows from (B.9) that $-\mu(\underline{c})\left[p \int_{\underline{c}}^{\underline{c}} H(\hat{c} \mid \underline{c}) \hat{c}+m^{\prime}(q(\underline{c}))\right]=0$. Therefore $q(\underline{c})=0$. This in turn implies that $m(q(\underline{c}))=m_{0}$ and so: $m(q(c, p))=m_{0}+\int_{\underline{c}}^{c} \zeta(t ; p) \mathrm{d} t$. But from (B.4) we can solve for $x(c)$ and substitute into (B.3). This yields

$$
R-m_{0}-\int_{\underline{c}}^{c} \zeta(t ; p) d t-v(c)+\dot{v}(c) c+q(c, p) p c \int_{\underline{c}}^{c} \frac{\partial H(\hat{c} \mid c)}{\partial c} \mathrm{~d} \hat{c}-q(c, p) p \int_{\underline{c}}^{c} H(\hat{c} \mid c) d \hat{c}=0
$$

and therefore

$$
\dot{v}(c) c-v(c)=-R+m_{0}+\int_{\underline{c}}^{c} \zeta(t ; p) \mathrm{d} t-q(c, p) p\left(c \int_{\underline{c}}^{c} \frac{\partial H(\hat{c} \mid c)}{\partial c} \mathrm{~d} \hat{c}-\int_{\underline{c}}^{c} H(\hat{c} \mid c) \mathrm{d} \hat{c}\right) .
$$

It follows that

$$
\begin{aligned}
v(c)=c K(R)-c \int_{\underline{c}}^{c} \frac{1}{s^{2}}\left(R-m_{0}-\int_{\underline{c}}^{s} \zeta(t ; p) \mathrm{d} t+\right. \\
\left.q(s, p) p s \int_{\underline{\underline{c}}}^{s} \frac{\partial H(\hat{c} \mid s)}{\partial s} \mathrm{~d} \hat{c}-q(s, p) p \int_{\underline{c}}^{s} H(\hat{c} \mid s) \mathrm{d} \hat{c}\right) \mathrm{d} s
\end{aligned}
$$

where $K(R)$ is a constant of integration which does depend on the parameter $R$. (B.4) now allows the characterization of $x(c)$.

This concludes the case of a binding transition equation and budget constraint.

Budget constraint not binding. The budget constraint is not binding and the ministry will have money left over if $\mu(\cdot)=0$. (B.6) implies a constant $\lambda(\cdot)$ for this case, while (B.8) implies that either $f(c)=\lambda=$ const in which case positive output may be produced, or output must be zero. This is due to the fact that it 
cannot be infinite since then the budget constraint would be binding. Suppose that output is positive: Then (B.9) indicates that the auditing probability should be increased (since the LHS of (B.9) is greater than zero) but as $q(\cdot)$ is increased it costs more money and ultimately the budget constraint must bind. Hence, positive output is incompatible with a slack budget. It follows that, if the budget is slack, output and auditing all are zero. Positive output and auditing therefore implies the budget constraint is binding and the non-binding condition is considered no further.

\section{Deriving the Ministry's Expected Payoff under Auditing}

Equation (7) in the text defines the ministry's (expected) payoff schedule $\mathrm{E}_{c}[u(c, R: A)]$. Differentiating (B.16) with respect to $c$ gives:

$$
\begin{gathered}
\dot{v}(c)=K(R)-\int_{\underline{c}}^{c} \frac{1}{s^{2}}\left(R-m_{0}-\int_{\underline{c}}^{s} \zeta(t ; p) \mathrm{d} t+q(s ; p) p s \int_{\underline{c}}^{s} \frac{\partial H(\hat{c} \mid s)}{\partial s} \mathrm{~d} \hat{c}-q(s ; p) p \int_{\underline{c}}^{s} H(\hat{c} \mid s) \mathrm{d} \hat{c}\right) \mathrm{d} s- \\
\frac{1}{c}\left(R-m_{0}-\int_{\underline{c}}^{c} \zeta(t ; p) \mathrm{d} t+q(c ; p) p c \int_{\underline{c}}^{c} \frac{\partial H(\hat{c} \mid c)}{\partial c} \mathrm{~d} \hat{c}-q(c ; p) p \int_{\underline{c}}^{c} H(\hat{c} \mid c) \mathrm{d} \hat{c}\right)
\end{gathered}
$$

Define $\chi(c, p)$ as:

$$
\chi(c, p)=\int_{\underline{c}}^{c} \frac{1}{s^{2}}\left(R-m_{0}-\int_{\underline{c}}^{s} \zeta(t ; p) \mathrm{d} t+q(s ; p) p s \int_{\underline{c}}^{s} \frac{\partial H(\hat{c} \mid s)}{\partial s} \mathrm{~d} \hat{c}-q(s ; p) p \int_{\underline{c}}^{s} H(\hat{c} \mid s) \mathrm{d} \hat{c}\right) \mathrm{d} s .
$$

Equation (8) follows immediately.

We are interested in the derivative of $\mathrm{E}_{c}[u(c, R: A)]$ with respect to $R$. $\partial \chi(c ; p) / \partial R=1 / \underline{c}-1 / c>0$. Also, since $v(\hat{r})=0$ if $\hat{r}<\bar{c}$ or $v(\bar{c})=0$ otherwise, we know that

$$
\begin{aligned}
0=v(\hat{r})=\hat{r} K(R)-\hat{r} \int_{\underline{c}}^{\hat{r}} \frac{1}{s^{2}}\left(R-m_{0}-\int_{\underline{c}}^{s} \zeta(t ; p) \mathrm{d} t+\right. \\
\left.\quad q(s, p) p s \int_{\underline{c}}^{s} \frac{\partial H(\hat{c} \mid s)}{\partial s} \mathrm{~d} \hat{c}-q(s, p) p \int_{\underline{c}}^{s} H(\hat{c} \mid s) \mathrm{d} \hat{c}\right) \mathrm{d} s .
\end{aligned}
$$

Taking the total derivative yields $0=\hat{r} \mathrm{~d} K-\hat{r} \int_{c}^{\hat{r}} s^{-2} \mathrm{~d} R \mathrm{~d} s$, and from that

$$
\frac{\mathrm{d} K}{\mathrm{~d} R}=\int_{\underline{c}}^{\hat{r}} \frac{1}{s^{2}} \mathrm{~d} s=-\frac{1}{\hat{r}}+\frac{1}{\underline{c}} .
$$

Taking the partial derivative of (8) we therefore obtain

$$
\frac{\partial \mathrm{E}_{c}[u(c, R: A)]}{\partial R}=\int_{\underline{c}}^{\hat{r}}\left(\frac{1}{\hat{r}}-\frac{1}{\underline{c}}+\frac{1}{\underline{c}}-\frac{1}{s}+\frac{1}{s}\right) f(s) \mathrm{d} s=\frac{1}{\hat{r}} F(\hat{r})>0
$$

which is equation (9) in the text. 


\section{The optimal reserve level of cost in an auction:}

The ministry's problem is

$$
\left.\max _{r} E_{c}[u(c, R, N: C)]=\int_{\underline{c}}^{r} \frac{R-b_{F}}{c} g_{(2)}(c) d c+\left[G_{(1)}(r)\right)-G_{(2)}(r)\right] \frac{R-b_{F}}{r} .
$$

This has first order condition:

$$
\frac{\left\{R-b_{F}\right\}\left(r g_{(1)}(r)-G_{(1)}(r)+G_{(2)}(r)\right)}{r^{2}}=0 .
$$

The second order condition for this problem is

$$
-\left(\frac{2\left[r g_{(1)}(r)-G_{(1)}(r)+G_{(2)}(r)\right]}{r}-\left(r g_{(1)}^{\prime}(r)+g_{(2)}(r)\right)\right) \frac{\left\{R-b_{F}\right\}}{r^{2}} \leq 0 .
$$

Using (D.2) in (D.3) implies that $r g_{(1)}^{\prime}(r)+g_{(2)}(r) \leq 0$. Expanding $g_{i}(\cdot)$, we obtain

$$
\begin{aligned}
r \frac{N !}{(N-1) !}\left(f^{\prime}(r)(1-F(r))^{N-1}-(N-2) f(r) f(r)(1-F(r))^{N-2}\right)+ & \\
\frac{N !}{(1) !(N-2) !} f(r) F(r)(1-F(r))^{N-2} & \leq 0
\end{aligned}
$$

and after simplification $r f^{\prime}(r)(1-F(r))-(N-2) f(r)(r f(r)+F(r)) \leq 0$. This in turn necessitates

$$
f^{\prime}(r)(1-F(r))-f(r)^{2} \leq(N-3) f(r)^{2}+(N-2) \frac{f(r) F(r)}{r} .
$$

In what follows, it is assumed that equation (D.5) holds. The first order condition (D.2) being satisfied implies

$$
\begin{array}{r}
r g_{(1)}(r)-\left(G_{(1)}(r)-G_{(2)}(r)\right)=0 \\
r N f(r)(1-F(r))^{N-1}-N F(r)(1-F(r))^{N-1}=0 \\
r f(r)-F(r)=0
\end{array}
$$

Using (D.6) in (D.5), the optimal reserve price $r^{*}$ satisfies:

$$
r^{*}=\frac{F\left(r^{*}\right)}{f\left(r^{*}\right)} \quad \text { when } \quad f^{\prime}\left(r^{*}\right)\left(1-F\left(r^{*}\right)\right) \leq 2(N-2) f\left(r^{*}\right)^{2} .
$$

For $r^{*}$ satisfying (D.7), the expected profits of the ministry then are

$$
\begin{aligned}
\mathrm{E}_{c}\left[u(c, R, N: C]=\int_{\underline{c}}^{r^{*}} \frac{R-b_{F}}{c} N(N-1) f(c) F(c)(1-F(c))^{N-2} \mathrm{~d} c+\right. & \\
& \frac{R-b_{F}}{r^{*}} N F\left(r^{*}\right)\left(1-F\left(r^{*}\right)\right)^{N-1} .
\end{aligned}
$$

Integrating by parts twice yields equation (14) in the text. 


\section{F. The effect of $N$ on the Marginal expected payoff from the auction.}

$$
\begin{aligned}
& \frac{\partial E_{c}[u(c, R, N: C)]}{\partial R}-\frac{\partial E_{c}[u(c, R, N-1: C)]}{\partial R}= \\
& \frac{1}{\underline{c}}-\frac{\left(1-F\left(r^{*}\right)\right)^{N}}{r^{*}}-\int_{\underline{c}}^{r^{*}} \frac{(1-F(c))^{N}}{c^{2}} \mathrm{~d} c-\int_{\underline{c}}^{r^{*}} \frac{N F(c)(1-F(c))^{N-1}}{c^{2}} \mathrm{~d} c-\frac{1}{\underline{c}}+ \\
& \quad \frac{\left(1-F\left(r^{*}\right)\right)^{N-1}}{r^{*}}+\int_{\underline{c}}^{r^{*}} \frac{(1-F(c))^{N-1}}{c^{2}} \mathrm{~d} c+\int_{\underline{c}}^{r^{*}} \frac{(N-1) F(c)(1-F(c))^{N-2}}{c^{2}} \mathrm{~d} c= \\
& F\left(r^{*}\right) \frac{\left(1-F\left(r^{*}\right)\right)^{N-1}}{r^{*}}-\int_{\underline{c}}^{r^{*}} F(c)^{2} \frac{(1-F(c))^{N-2}}{c^{2}} \mathrm{~d} c+\int_{\underline{c}}^{r^{*}} \frac{N F(c)^{2}(1-F(c))^{N-2}}{c^{2}} \mathrm{~d} c= \\
& F\left(r^{*}\right) \frac{\left(1-F\left(r^{*}\right)\right)^{N-1}}{r^{*}}+\int_{\underline{c}}^{r^{*}} \frac{(N-1) F(c)^{2}(1-F(c))^{N-2}}{c^{2}} \mathrm{~d} c>0 .
\end{aligned}
$$

\section{REFERENCES}

Alesina, A., Wacziarg, R., 1998. Openness, country size and government. Journal of Public Economics. 69: $305-321$.

Andreoni, J., 1991. Reasonable doubt and the optimal magnitude of fines: should the penalty fit the crime? Rand Journal of Economics. 22( 3), 385-395.

Baron, David P., Besanko, David, 1984. Regulation, Asymmetric Information and Auditing. The Rand Journal of Economics, 15(4): 447-470.

Bendor, J., Taylor, S., Van Gaalen, R., 1985. Bureaucratic expertise versus legislative authority: A model of deception and monitoring in budgeting. The American Political Science Review. 79: 1041-1060.

Bose, P., 1995. Regulatory errors, optimal fines and the level of compliance. Journal of Public Economics. 56: $475-484$.

Breton, A., Wintrobe, R., 1975. The equilibrium size of a budget-maximizing bureau: A note on Niskanen's theory of bureaucracy. Journal of Political Economy. 83(1): 195-207.

David, H.A., 1981. Order Statistics, 2nd Edition, John Wiley and Sons Inc., New York, USA.

Davis, L.D., Hayes, K, 1993. The Demand for Good Government. The Review of Economics and Statistics. 75(1): 148-152.

Dickens, W.T., Katz, L.F., Lang, K., Summers, L. H., 1989. Employee crime and the monitoring puzzle. Journal of Labor Economics. 7(3): 331-347. 
De Fraja, G., 1993. Productive efficiency in public and private firms. Journal of Public Economics. 50: $15-30$.

Feenstra, R.C., Hanson,G. H., 1996. Globalization, outsourcing and wage inequality. The American Economic Review. 86(2): 240-245.

Hart, O., Shleifer, A., Vishny, R.W., 1997. The proper scope of government: Theory and an application to prisons. The Quarterly Journal of Economics. 112(4): 1127-1161.

Hettich, W., Winer, S.L., 1988. Economic and political foundations of tax structure. The American Economic Review. 78(4): 701-712.

Hogg, Robert V., Craig, T. Allen, 1978. Introduction to Mathematical Statistics, fourth edition. Macmillan Publishing Co., Inc., New York, USA.

Holsey, Cherly M., Borcherding, Thomas E., 1997. Why does government's share of national income grow? An assessment of the recent literature on the U.S. experience. Perspective on Public Choice: A Handbook. Edited by Mueller, Dennis C., Cambridge University Press, United Kingdom.

Jehle, A. Geoffrey, Reny, Philip J., 2001. Advanced Microeconomics Theory, second edition. Addison Wesley. Toronto, Canada.

Jewitt, Ian, 1988. Justifying the first-order approach to principal-agent problems. Econometrica. 56(5): $1177-1190$.

Kamien, Morton I., Schwartz, Nancy L., 1981. Dynamic Optimization: The calculas of variations and optimal control in economics and management, Series volume 4, North-Holland, New York

Lacker, Jeffrey M., Weinberg, John A., 1989. Optimal contracts under costly state falsification. Journal of Political Economy. 97(6): 1345-1363.

Laffont, J.J., Tirole, J., 1986. Using cost observation to regulate firms. Journal of Political Economy. 94:3: $614-641$.

McAfee, P.P., McMillan, J., 1987. Auctions and bidding. Journal of Economic Literature. XXV(6): 699-738.

Milgrom, Paul R., 1987. Auction Theory in Truman Bewley (ed.), Advances in Economic Theory —Fifth World Congress, Cambridge University Press,Cambridge, England. 
Mookherje, D., Png, I.P.L., 1992. Monitoring vis-a-vis investigation in enforcement of law. The American Economic Review. 82(3): 556-565.

Mueller, D.C., 1997. Perspectives on Public Choice: A Handbook. Cambridge University Press, United Kingdom.

Niskanen, W.A., 1975. Bureaucrats and politicians. Journal of Law and Economics. XVIII (3), 217-243.

Osborne, D., Gaebler, T., 1993. Reinventing Government: How the Entrepreneurial Spirit is Transforming the Public Sector. Penguin Books USA Inc., New York.

Savas, E.S., 1977. Policy analysis for local government: Public vs private refuse collection. Policy Analysis. 3(1), 49-74.

Rogerson, William P., 1985. The first-order approach to principal-agent problems. Econometrica. 53(6): $1357-1367$.

Stevens, B.J., 1978. Scale, market structure, and the cost of refuse collection. Review of Economics and Statistics. 60: 395-406.

ECONOMICS, YORK UNIVERSITY

E-mail address: Gervan.Fearon@yorku.ca

Department of Economics, University of Waterloo, Waterloo ON N2L 3G1

E-mail address: lbusch@uwaterloo.ca 